\title{
Numerical aspects of nonreinforcement: The same-phase nonreinforcement procedure
}

\author{
E. J. CAPALDI, DANIEL J. MILLER, and SUZAN ALPTEKIN \\ Purdue University, West Lafayette, Indiana
}

\begin{abstract}
To test the hypothesis that distinctive internal representations are associated with number of successively presented nonreinforcements, four investigations employed rats in a discrete trial runway task (go/no-go), in which the smaller number of nonreinforcements was a signal for reinforcement (S + cue), the larger number of nonreinforcements was a signal for nonreinforcement (S- cue). Consistent with the hypothesis under test, discriminative responding increased as the numerical difference between the $S+$ and $S-$ cues increased. These results are highly similar to those previously obtained employing the conventional extinction procedure. However, unlike extinction data, the present findings cannot be interpreted without reference to numerical representations because better experimental control was employed here than has been in conventional extinction investigations. It was suggested that rats are capable of enumerating, or counting, successive nonreinforcements and that the tendency of rats to enumerate all types of reinforcing events is of importance for understanding not merely extinction, but a wide array of learning phenomena.
\end{abstract}

Conventional extinction investigations employ the "uncle" procedure, in that they follow an initial acquisition phase in which responding grows vigorous with a long series of nonreinforcements that are administered until the animal gives up vigorous responding - in effect, crying uncle. Consider any nonreinforced trial in a series of nonreinforcements, say the 10th. It occurs following nine prior nonreinforcements (number cue), it occurs later in the series than each of the prior nine nonreinforcements (time cue), and it occupies a particular position in the series, the 10th position (position cue). Thus, the uncle procedure completely confounds cues recently suggested to be of considerable importance, number cues (e.g., Capaldi \& Miller, 1988a; Church \& Meck, 1984), time cues (e.g., Meck \& Church, 1983), and position cues (e.g., Burns, Wiley, \& Payne, 1986; Roitblat, Polage, \& Scopatz, 1983; Terrace, 1986). With the uncle procedure, the value of number, time, and position cues will consistently increase and, at some point in extinction, so exceed the value that signaled reinforcement in acquisition that responding will necessarily decline in vigor. However, to what extent, if any, each of the three classes of aforementioned cues contribute to the decline in responding can hardly be determined on the basis of the uncle procedure. In the four investigations reported here, we attempted to determine if distinctive numerical cues are associated with successively presented nonreinforcements. We employed a novel go/no-go discrimination learning procedure that was closely modeled after extinction investigations, and that overcame the limitations of

This research was supported in part by Grant BNS-8515831 from the National Science Foundation to E. J. Capaldi. Correspondence may be addressed to E. J. Capaldi, Department of Psychological Sciences, Purdue University, West Lafayette, IN 47907. the uncle procedure by allowing us to unconfound number of events from positional and temporal cues, since we were not evaluating the role of these latter cues in the present investigations. Our only concern with position and time cues was to eliminate them.

We wanted to test the following assumptions. Various reinforcement events contingent upon instrumental responding possess attributes (e.g., sweetness, hardness, number) that may be stored in memory. Such attributes may be retrieved on some subsequent trial provided the retrieval trial is sufficiently similar to the storage trial (e.g., Capaldi, 1971; Capaldi, Verry, Nawrocki, \& Miller, 1984; Jobe, Mellgren, Feinberg, Littlejohn, \& Rigby, 1977). Retrieved attributes become signals for the reinforcing event that occurs on the retrieval trial, and thus such attributes function as an anticipatory mechanism (Capaldi \& Miller, 1988b; Capaldi, Nawrocki, \& Verry, 1983). Vigor of responding is an increasing function of the magnitude of reinforcement signaled by the retrieved attributes. Among the attributes common to all reinforcers are numerical ones, distinctive internal representations that are associated with number of successively presented reinforcing events. Let us apply these assumptions to conventional extinction investigations.

In the acquisition phase of extinction investigations, representations corresponding to small numbers of nonreinforcement become $S+$ cues, signaling reinforcement either indirectly, through generalization from other reinforcers, or directly, under partial reinforcement, for example, when one or more nonreinforced trials is followed by a reinforced trial (e.g., Capaldi, 1966). Representations corresponding to large numbers of nonreinforcements become $\mathrm{S}$ - cues, signaling nonreinforcement because of the long strings of nonreinforced trials provided in the subsequent extinction phase. According to this anal- 
ysis, extinction investigations are an unorthodox form of discrimination learning task in which $\mathrm{S}+$ cues are presented in the initial phase, called the acquisition phase, and $S$ - cues are presented in a subsequent separate phase, called the extinction phase. The four investigations reported here may be characterized as relatively orthodox discrimination learning investigations that are, nevertheless, closely modeled after extinction. Rather than presenting the numerical $\mathbf{S}+$ and $S-$ cues in separate phases, as in extinction investigations, we presented the $\mathrm{S}+$ and $\mathrm{S}-$ cues in the same phase, as is typical of discrimination learning studies in general. The same-phase nonreinforcement procedure allows better control over number cues than does the separate-phase procedure.

It is assumed that the similarity between nonreinforced cues decreases as the numerical difference between them increases. In some investigations reported here, the similarity between the $S+$ and $S$ - cues was greater than in others. It was expected that discriminative responding would increase along with an increase in dissimilarity between the $S+$ and $S$ - cues. More particularly, when the $S$ - cue was a given number of nonreinforcements, discriminative responding would tend to decrease as the $S+$ cue became more similar to the $S-$ cue. This principle, of course, is identical to that which would be applied to any other stimulus dimension. The important point to be underlined here is this: The conclusion that number of nonreinforcements give rise to discriminable cues can be reached only by comparing the results obtained in all four investigations reported here. This being the case, the results of all four investigations will be presented simultaneously for easy comparison.

In each group designation, two numbers are used. The first refers to the smaller number of nonreinforcements used as the $\mathbf{S}+$ cue, the second refers to the larger number used as the $\mathrm{S}-$ cue. For example, the designation 1-2 means that a single nonreinforced trial was the $S+$ cue, because it was followed on $50 \%$ of the occasions by a reinforced trial, and two nonreinforced trials were the $\mathrm{S}-$ cue, because they were always followed by a nonreinforced trial. In Group 1-2, the appropriate discriminative responding, were it to occur, would be slower running following two nonreinforced trials than following one nonreinforced trial. The other three groups were $0-2$, 1-3, and 2-3.

\section{EXPERIMENTS 1-4}

\section{Method}

\section{Subjects}

All 13 subjects were experimentally naive male albino rats (Holtzman Co., Madison, Wisconsin). On arrival at the laboratory, all rats, which were about 77 days old, were placed in individual cages and provided with food and water ad lib for either 15 days, Group 1$2(n=3)$ and Group 2-3 $(n=4)$, or 35 days, Group 0-2 $(n=3)$ and Group 1-3 $(n=3)$, prior to pretraining.

\section{Apparatus}

A straight gray alley runway was used in all four experiments. It measured $194.90 \mathrm{~cm}$ in length, $8.8 \mathrm{~cm}$ in width, with sides
$14.0 \mathrm{~cm}$ high. The entire alley was covered by wire mesh on a hinged frame. The initial $23.3 \mathrm{~cm}$ and the last $36.15 \mathrm{~cm}$ were closed off by metal guillotine doors to form the startbox and goalbox, respectively. Raising the startbox door triggered a microswitch that started an electronic $0.01-\mathrm{sec}$ clock. The clock stopped when the rat broke a photobeam $158.13 \mathrm{~cm}$ beyond the startbox door and $6 \mathrm{~cm}$ in front of a rectangular $6.5 \times 8.8 \times 3.8-\mathrm{cm}$ wooden block at the back of the goalbox. Cut into the block was a circular food cup, $4 \mathrm{~cm}$ in diameter and $1.5 \mathrm{~cm}$ in depth, in which food pellets were placed on reinforced trials. To time confinement duration, a second electronic 0.01 -sec clock started simultaneously with the stopping of the first clock. To prevent retracing, the goalbox door was lowered when the rat broke the photobeam.

\section{Design}

Runway trials terminated with $15 \mathrm{sec}$ of confinement in the goalbox, where rats received either reinforcement $(R)$, six .045-g Noyes pellets, or nonreinforcement $(N)$, which consisted simply of confinement to the unbaited goalbox.

Four or five runway trials in succession define a series. In the four-trial series RRRN, for example, each of the first three trials terminates in $R$, the fourth, and last, trial of the series is N. Thus, Positions 1, 2, and 3 of the RRRN series are associated with $R$; Position 4 is associated with $N$. Intervals between trials were spent in a waiting cage in the experimental room. Table 1 shows that each of the four groups received two types of series: an $R$ series, in which most trials terminated in $\mathrm{R}$, and an $\mathrm{N}$ series, in which all trials following the first terminated in $\mathrm{N}$. Trials of a series were separated by an interval of about $30 \mathrm{sec}$; series presentations were separated by an interval of about $10-15 \mathrm{~min}$. These temporal intervals were used to enable us to run the rats in squads, giving each rat all the trials of its series before the next rat was run. On each day of experimental training, each rat received three presentations of the $R$ series and three of the $N$ series. Group $0-2$ received only one type of $R$ series; each of the remaining three groups received two slightly different types of $\mathbf{R}$ series. Our purpose in this study was to demonstrate that rats can discriminate among number of successively presented $\mathrm{N}$ trials when percentage of reinforcement, position cues, and time cues are controlled. Below, we consider each of these variables in relation to the training shown in Table 1.

Number of nonreinforcements. The first and second numbers in the group designations shown in Table 1 refer to the number of successive $\mathrm{N}$ trials that became signals for reinforcement $(R$ series) and nonreinforcement ( $\mathrm{N}$ series), respectively. Consider, for example, Group 1-2. In both of its $\mathrm{R}$ series, a single $\mathrm{N}$ trial was always followed by $\mathbf{R}$, and so became a signal for $\mathbf{R}(\mathrm{S}+$ cue); in its $\mathrm{N}$ series, two successive $\mathrm{N}$ trials were always followed by $\mathbf{N}$ ( $\mathrm{S}-$ cue), and so became a signal for $\mathbf{N}$. If distinctive cues are associated with number of $\mathbf{N}$ event, then the greater the difference between the $S+$ and $S-$ cues, the greater should be the tendency for running to be slower in the $\mathrm{N}$ series than in the $\mathrm{R}$ series. Thus,

Table 1

The $\mathbf{R}$ Series and the $\mathbf{N}$ Series Received by Each Group

\begin{tabular}{|c|c|c|c|}
\hline \multirow[b]{3}{*}{ Group } & \multicolumn{2}{|c|}{ R Series } & \multirow{2}{*}{$\frac{\text { N Series }}{\text { Position }}$} \\
\hline & Position & Position & \\
\hline & 12345 & 12345 & 12345 \\
\hline $\begin{array}{l}0-2 \\
1-2 \\
1-3 \\
2-3\end{array}$ & $\begin{array}{lllll}\mathbf{R} & \mathbf{R} & \mathbf{R} & \mathbf{N} \\
\mathrm{R} & \mathbf{N} & \mathbf{R} & \mathbf{R} \\
\mathrm{R} & \mathbf{N} & \mathbf{R} & \mathbf{R} & \mathbf{R} \\
\mathrm{R} & \mathbf{N} & \mathbf{N} & \mathbf{R} & \mathbf{R}\end{array}$ & 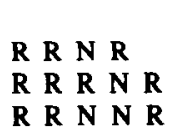 & $\begin{array}{lllll}\mathbf{R} & \mathbf{N} & \mathbf{N} & \mathbf{N} \\
\mathbf{R} & \mathbf{N} & \mathbf{N} & \mathbf{N} \\
\mathbf{R} & \mathbf{N} & \mathbf{N} & \mathbf{N} & \mathbf{N} \\
\mathbf{R} & \mathbf{N} & \mathbf{N} & \mathbf{N} & \mathbf{N}\end{array}$ \\
\hline
\end{tabular}

Note- $R$ and $N$ signify reinforcement and nonreinforcement, respectively. Four or five trials in succession define a series. In the four-trial series RRRN, for example, each of the first three trials terminates in $R$, the fourth, or last, trial of the series in N. From a positional perspective, then, Positions 1, 2, and 3 of the RRRN series are associated with $R$, and Position 4 is associated with $N$. 
Group 0-2 should show a greater tendency toward discriminative responding than Group 1-2, and Group 1-3 should show a greater tendency toward discriminative responding than Group 2-3. In Group 0-2, the $\mathrm{N}$ trial that terminated the $\mathrm{R}$ series was separated from prior $R$ trials by a 30 -sec interval and from a subsequent $R$ trial (the first $R$ trial of the next series) by about 10-15 min. Prior evidence shows that under these conditions an $\mathrm{N}$ trial will not become a signal for an $R$ trial, hence the $S+$ cue for Group $0-2$ is listed as 0 (see Capaldi, Nawrocki, Miller, \& Verry, 1986).

Percentage of reinforcement. Suppose that speed of running in the $\mathbf{N}$ series would decrease as percentage of reinforcement in the $R$ series increases. If this were the case, Groups 0-2 and 1-2 should not differ. These groups receive the same reinforcement percentages in both series (75\% in the $\mathrm{R}$ series, $25 \%$ in the $\mathrm{N}$ series).

Position cues. Considering the $\mathrm{R}$ series and the $\mathrm{N}$ series received by each group, each position has associated with it a particular reinforcement percentage that should determine completely vigor of responding at that position. There is, however, no basis in the position view for expecting that performance at a particular position should be differential with respect to type of series ( $R$ series vs. $\mathbf{N}$ series). For example, if only positional information was utilized, Group 0-2 should nun slowly on Position 4 of both series, since that position always is nonreinforced. Only one other position received consistent nonreinforcement, Position 3 in Group 2-3. Reinforcement percentages at other positions range from $33 \%$ to $100 \%$. If vigor of responding decreases as percentage of reinforcement of a particular position decreases, the position view would be confirmed.

Time. When timing in the run mode, an initial stimulus starts the timing process, which continues until some subsequent event (Church \& Meck, 1984). In the present case, this means that the rats would time response durations, intertrial intervals, and goalbox confinement times on $\mathrm{R}$ and $\mathrm{N}$ trials, starting with some event. This view encounters many of the same difficulties as the position view considered above. Thus, considering the $R$ series and the $N$ series received by each group, each temporal cue has associated with it a particular reinforcement percentage that should determine completely vigor of responding. Response vigor, therefore, should not be differential with respect to series. Consider why this is so, in relation to Group 1-3. In each series, the time elapsing from the first trial to the subsequent trial is the same. Thus, an animal employing this temporal information could not respond differentially with respect to series. This would also be the case if run timing were begun with the first $\mathbf{N}$ trial of each series. However, to suggest that run timing (or marking positions) were marked off from the second trial of the $N$ series of Group 1-3 would be to accept the number view, which holds that rats can discriminate among nonreinforcements on the basis of number.

When timing in the stop mode, animals are timed only during the presentation of the stimulus (Church \& Meck, 1984). If, for example, Group 1-3 timed $N$ trials in the stop model, then 15-sec $\mathrm{N}$ confinement would be an $\mathrm{S}+$ cue and $45-\mathrm{sec} \mathrm{N}$ confinement, an $S-$ cue. To evaluate this possibility, we did the following: After 10 days of experimental training, Group 1-3 received 6 days of shift, in which it was trained as before, but with one exception. On the $\mathbf{N}$ trials of its two $\mathbf{R}$ series, confinement duration was increased to $45 \mathrm{sec}$, but remained at $15 \mathrm{sec}$ on the $N$ trials of its $N$ series. This should have eliminated discriminative responding when the rats were timed in the stop mode during $\mathrm{N}$ trials.

\section{Procedure}

There were a total of five experimenters, one each for Groups 0-2, 1-2, and 1-3 and two for Group 2-3. Each of the two experimenters in Group 2-3 ran 2 rats. Beginning on Day 1 of pretraining, the rats were placed on a deprivation schedule of $15 \mathrm{~g}$ of Wayne Lab Blox minus any food eaten in the apparatus. On Days 1-11, the rats were handled for about $1 \mathrm{~min}$ each. Alley exploration was car- ried out on Days 12-14. On each of these days, each rat was fed six .045-g Noyes pellets in the home cage, and then was given $3 \mathrm{~min}$ of alley exploration. Six pellets were scattered about the runway. To explain how series were presented to the rats, we have designated them by letters. The series received by Group $0-2$ were $(A)$ RRRN and (B) RNNN; by Group 1-2, (A) RNRR, (B) RRNR, and (C) RNNN; by Group 1-3, (A) RNRRR, (B) RRRNR, and (C) RNNNN; by Group 2-3, (A) RNNRR, (B) RRNNR, and (C) RNNNN. On each of 3 days prior to experimental training, Group 02 received its A series three times. On Day 12 of pretraining, groups 1-2 and 2-3 received the $A$ series; on Day 13, the $C$ series, then the $B$ series; and on Day 14, the A, B, and $C$ series, in that order. On Day 12 of pretraining, Group 1-3 received its A series, then its B series; on Day 13, its B series, then its A series; on Day 14, its $A B B A A B$ series, in that order; and on Day 15, its BAABBA series, in that order. Following the last trial of the day in pretraining, as in experimental training, the rats were returned to the home cage, where they were fed the daily ration about $15 \mathrm{~min}$ later.

In experimental training, all rats to be run by a particular experimenter were brought into the experiment room from the colony room. Each rat received all the trials of a series before the next rat received its series. This produced an intertrial interval of about $30 \mathrm{sec}$ and an interseries interval of about 10-15 min. The order of running the rats was varied daily. Each rat was allowed a maximum of $60 \mathrm{sec}$ to enter the goalbox. If the rat had not entered the goalbox within $60 \mathrm{sec}$, it was placed there and given the scheduled event (either $\mathrm{R}$ or $\mathrm{N}$ ). Each received six series presentations each day, three Rs and three Ns. Over a 2-day block, each of the two $R$ series received by Groups 1-2, 1-3, and 2-3 were presented equally often. On Days 1-4, the order of presenting the series for Groups $1-2,1-3$, and 2-3 was BCACCB, CACBAC, ACBCCA, and CBCABC. These orders were repeated in each subsequent 4-day block. Group 1-2 was run for 38 days, Group 2-3 for 34 days, and Group 1-3 for 10 days. Group 0-2, also run for 10 days, received its series in the order $A B B A A B$ on odd days and in the order BAABBA on even days. Groups 1-2 and 2-3 were run until it became reasonable to assume that they were not going to respond discriminatively following different numbers of nonreinforcements or at least that discriminative responding in these groups was greatly retarded relative to that in Groups $0-2$ and 1-3.

After 10 days of experimental training, Group 1-3 received 6 days of shift, in which it was trained as in experimental training, but with one exception. On the $\mathrm{N}$ trials of its two $\mathrm{R}$ series, confinement duration was increased to $45 \mathrm{sec}$, but remained at $15 \mathrm{sec}$ on the $\mathbf{N}$ trials of its $\mathbf{N}$ series.

\section{Results}

In Groups 1-2, 2-3, and 1-3, each $R$ series occurred half as often as the $\mathrm{N}$ series. Thus, for Groups 1-2, 2-3, and 1-3, an analysis of variance was performed over the trial means of each $\mathbf{R}$ series separately and over the $\mathbf{N}$ trials only of the $\mathbf{N}$ series. For Group $0-2$, the analysis was performed over the raw speeds of the $R$ series and the $\mathbf{N}$ trials of the $\mathbf{N}$ series. The initial $\mathbf{R}$ trial of each $\mathbf{N}$ series was omitted from the analysis because it obviously was not an S- trial. In all four experiments, the discriminative responding was progressively slower running over successive $\mathbf{N}$ trials of the $\mathbf{N}$ series. Within the first few days of training, discriminative responding appeared in Groups 0-2 and 1-3, thus their training was either terminated after 10 days (Group 0-2) or modified (Group 13). Not only did Groups 1-2 and 2-3 fail to respond discriminatively over the first few days of training, they failed to do so over any of the days they were trained (38 
days and 34 days, respectively). For Groups 1-2 and 2 3 , we report results for the first 10 days of training and the last 10 days of training. These results are quite representative of all training days.

A difference will be said to be significant only if $p<.05$ or better. Figure 1 shows the mean speeds of each group on each trial of their respective $R$ series (left panel) and on each $\mathbf{N}$ trial of their respective $\mathbf{N}$ series (right panel). For Groups 1-2 and 2-3, these means are shown for the first five blocks of training (10 days) and the last five blocks. The means for Groups 0-2 and 1-3 over the five blocks shown in Figure 1 constituted the totality of training received by these groups in original training. Although each of the two $R$ series received by Groups 1-2, 2-3, and 1-3 were analyzed separately, we combined them for purposes of presentation in Figure 1. In each group, the speeds on the initial $\mathbf{R}$ trials of the $\mathbf{N}$ series (not shown in Figure 1) were practically identical to those on the initial $\mathbf{R}$ trials of the $\mathbf{R}$ series. Considering all four groups, only in two instances were differences associated with the R series significant. Over the first five blocks, differences associated with the B series only of Groups $1-2$ and $2-3$ were significant $[F(3,6)=8.41$ and $F(4,12)$ $=3.85$, respectively]. For both of these groups, there were no other significant differences in either the $R$ or the $\mathrm{N}$ series. As may be seen in Figure 1, Groups 1-2 and $2-3$, if anything, ran faster on both their $R$ and $N$ se-

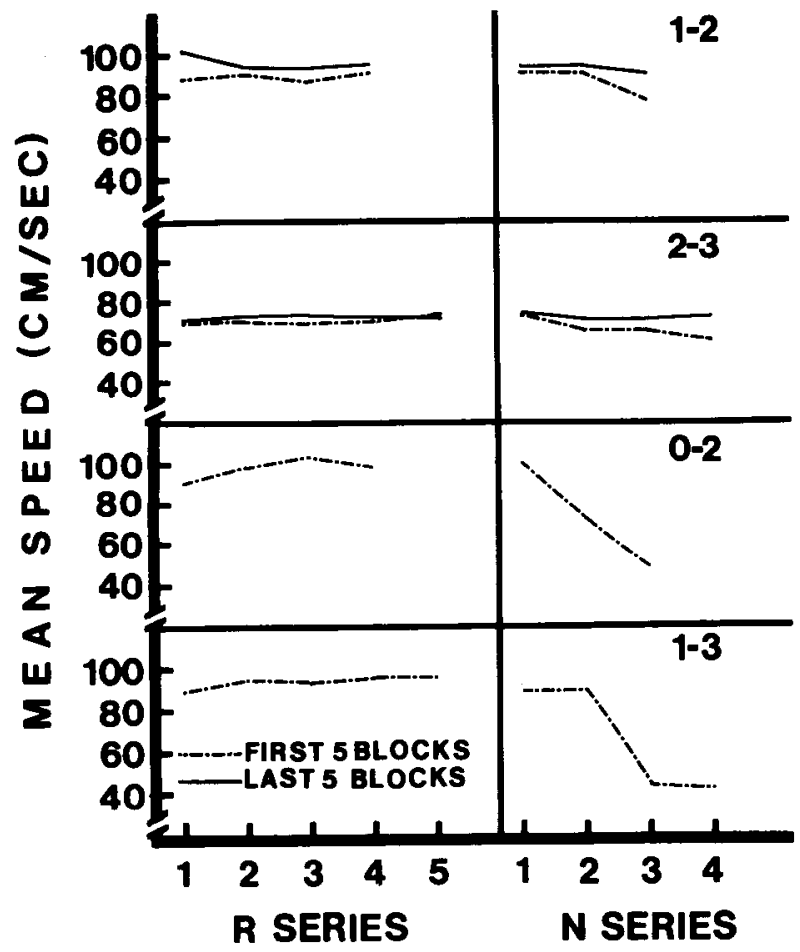

Figure 1. Mean speed for each group on each trial of their respective $R$ series (left panel) and on each $\mathbf{N}$ trial of their respective $\mathbf{N}$ series (right panel). For Groups 1-2 and 2-3, these means are shown in blocks of two days for the first five blocks of training and the last five blocks of training. For Groups 0-2 and 1-3, the means shown are for the first five blocks of training. ries as training progressed, which indicated that discriminative responding was not about to occur. In the $\mathrm{N}$ series of Group 0-2, significant differences were associated with trials $[F(2,4)=104.07]$, and no other difference was significant. In the $N$ series of Group 1-3, significant differences were associated with trials $[F(3,6)=$ $18.01]$ and the trials $\times$ blocks interaction $[F(4,8)=4.06]$. A breakdown of the significant trials effect employing the Newman-Keuls test indicated that in Group 1-3, Trial 3 differed significantly from Trials 1 and 2 , as did Trial 4, and no other difference was significant.

Increasing confinement duration to $45 \mathrm{sec}$ on the $\mathrm{N}$ trials of $R$ series received by Group 1-3 had no effect on responding in either their $\mathrm{R}$ series or their $\mathrm{N}$ series. When the shift speeds of Group 1-3 are plotted in 2-day blocks, on each of the three blocks, they resemble entirely the speeds on the $\mathrm{R}$ and $\mathrm{N}$ series shown in Figure 1 for Group 1-3. There were no significant differences associated with the $\mathbf{R}$ series on any of the three blocks. However, on each of the three blocks, significant differences were associated with trials $[F(3,6)=20.26,23.28$, and 19.85 , respectively]. No other differences were significant. The Newman-Keuls tests indicated that, as in original training, on each block, Trial 3 differed significantly from Trials 1 and 2 , as did Trial 4 , and no other difference was significant.

\section{GENERAL DISCUSSION}

Groups 0-2 and 1-3 showed discriminative responding following minimal training, while Groups 1-2 and 2-3 did not, even after extensive training. Furthermore, increasing confinement duration on nonreinforcement trials of the $R$ series of Group 1-3 had no effect on established discriminative responding. These findings are consistent with the view that discriminable cues are associated with number of successively presented nonreinforcements. However, the findings obtained here cannot be explained in terms of percentage of reinforcement, position cues, or time cues. A percentage approach cannot explain better discriminative responding by Group 0-2 than by Group 1-2. Discriminative responding (e.g., slower running by Group 1-3 in its $N$ series than in its $R$ series) cannot be explained by a position- or time-cue approach. Responding also cannot be understood in terms of the reinforcement percentage that occurs in connection with a particular positional or temporal cue. For example, in Group 2-3, the third trial of each of its three series terminated in nonreinforcement, yet running was rapid on that trial in each series.

It has been shown that, with the uncle procedure, as the number of nonreinforcements that become signals for reinforcement in the acquisition phase increase, subsequent resistance to extinction also increases, even when the interval between trials is as long as $24 \mathrm{~h}$. This rules out fading trace type explanations (see, e.g., Capaldi, 1967; Capaldi et al., 1986; Jobe et al., 1977). In other words, results obtained employing the separate-phase ex- 
tinction procedure are similar to those obtained here employing the same-phase nonreinforcement procedure, which implicate long-term memory. However, better experimental control was employed here than has been in conventional extinction investigations. Thus, while the present findings cannot be explained without reference to number of nonreinforcements, the uncle findings, as indicated, may be explained in terms of number, time, and/or position cues associated with nonreinforcements. Certainly, the present results are consistent with the view that number cues associated with nonreinforcements may be an important variable under the uncle procedure (see, e.g., Capaldi, 1967). It would be helpful to determine if the same is true for time and position cues, when the uncle procedure is employed.

If counting is defined as discriminating among events on the basis of their number (e.g., Church \& Meek, 1984), then rats in the present investigations counted nonreinforcements. A more stringent, or at least different, definition of counting is that the numerical discrimination must be shown to involve abstraction, cardinality, and these three principles: one to one correspondence, order irrelevance, and stable order (see, e.g., Davis \& Memmott, 1982; Davis \& Perusse, in press). According to this definition, counting was not demonstrated here. However, it recently has been shown that successively presented food reinforcements are counted by rats, according to the more stringent definition (Capaldi \& Miller, 1988a). It seems reasonable to assume that an animal capable of counting successively presented food reinforcements is not only capable of counting successively presented nonreinforcements, but that it normally will do so when such number cues are of predictive usefulness, as in the present investigations. Capaldi and Miller (1988a) also demonstrated that when number of food reinforcements is confounded with various other cues, rats will nevertheless count the food reinforcers (see also, Church \& Meck, 1984, for similar findings). Of course, in many conventional instrumental learning investigations, number of nonreinforcements and other cues normally are confounded, as, for example, in the uncle procedure (see, e.g., Capaldi, 1967; Haggbloom, 1982). Given the findings of Capaldi and Miller (1988a), then, it is not unreasonable to suggest that when number cues associated with nonreinforcements are confounded with other cues, rats may nevertheless enumerate nonreinforcements. This being so, it follows that animals count when other means of solution are available and that counting reinforcing events may be employed to understand a wide array of learning phenomena (see, e.g., Capaldi, 1964, 1966, 1967; Capaldi \& Miller, 1988a, 1988b).

Having examined the animal counting literature, Davis and Memmott (1982) concluded that animals discriminate among number of events only with great difficulty, and so counting is a strategy employed by animals only as a last resort when no other solution is available. Accepting that the literature available to Davis and Memmott is generally correct, it certainly cannot be claimed that our Groups 0-2 and 1-3 had any difficulty discriminating among number of events. These groups formed the numerical discrimination rapidly, we suggest, because they were provided with an easy discrimination learning problem. Perhaps most of the numerical discrimination learning problems surveyed by Davis and Memmott were difficult ones. One possible conclusion, then, is that discrimination learning problems involving counting are similar to other discrimination learning problems, in that the emergence of discrimination responding depends upon the similarity of the $S+$ and $S-$ cues. This view, which is consistent with the present findings, is also consistent with data reported by Hicks (1956). Another possible conclusion, one more congenial to Davis and Memmott (1982) perhaps, is that while Groups 0-2 and 1-3 solved their discrimination learning problem with relative ease, they were employing some numerical ability simpler than counting-for example, a few-many discrimination (Davis $\&$ Perusse, 1988). With this view, it is only discrimination learning problems involving true counting that invariably are difficult to solve by animals. However, our own preference is to assume, if only tentatively, that rats, having shown themselves adept at counting successively presented food reinforcements (Capaldi \& Miller, 1988a), would in all probability have counted successively presented nonreinforcements in the present investigations.

\section{REFERENCES}

Burns, R. A., Wiley, L. P., Payne, T. L. (1986). Temporal cuing of runs in series of reward events reduces interevent anticipation. Animal Learning \& Behavior, 14, 190-196.

CAPALDI, E. J. (1964). Effect of N-length, number of different $\mathrm{N}$-lengths, and number of reinforcements on resistance to extinction. Journal of Experimental Psychology, 68, 230-239.

CAPALDI, E. J. (1966). Partial reinforcement: A hypothesis of sequential effects. Psychological Review, 73, 459-477.

CAPALDI, E. J. (1967). A sequential hypothesis of instrumental learning. In K. W. Spence \& J. T. Spence (Eds.), The psychology of learning and motivation: Advances in research and theory (Vol. 1, pp. 67156). New York: Academic Press.

CAPALDI, E. J. (1971). Memory and learning: A sequential viewpoint. In W. K. Honig \& H. James (Eds.), Animal memory (pp. 115-154). New York: Academic Press.

Capaldi, E. J., \& Miller, D. J. (1988a). Counting in rats: Its functional significance and the independent cognitive processes which comprise it. Joumal of Experimental Psychology: Animal Behavior Processes, 14, 3-17.

CAPAldi, E. J., MiLler, D. J. (1988b). The rat's simultaneous anticipation of remote events and current events can be sustained by event memories alone. Animal Learning \& Behavior, 16, 1-7.

Capaldi, E. J., Nawrocki, T. M., Miller, D. J., \& Verry, D. R. (1986). Time between events as a retrieval cue: Recall and the temporal similarity between the storage and retrieval intervals. Journal of Experimental Psychology: Animal Behavior Processes, 12, 258-269.

CaPaldi, E. J., NawrockI, T. M., VerRY, D. R. (1983). The nature of anticipation: An inter- and intraevent process. Animal Learning \& Behavior, 11, 193-198.

Capaldi, E. J., Verry, D. R., Nawrocki, T. M., Miller, D. J. (1984). Serial learning, interitem associations, phrasing cues, interference, overshadowing, chunking, memory, and extinction. Animal Learning \& Behavior, 12, 7-20. 
Church, R. M., Meck, W. H. (1984). The numerical attribute of stimuli. In H. L. Roitblat, T. G. Bever, \& H. S. Terrace (Eds.), Animal cognition (pp. 445-464). Hillsdale, NJ: Erlbaum.

DAvis, H., MemmotT, J. (1982). Counting behavior in animals: A critical evaluation. Psychological Bulletin, 92, 547-571.

Davis, H., Perusse, R. (in press). Numerical competence in animals: Definitional issues, current evidence, and a new research agenda. $B e$ havioral \& Brain Sciences.

НАGGвLоOм, S. J. (1982). Effect of N-R transitions during partial reinforcement pretraining on subsequent resistance to discrimination. Animal Learning \& Behavior, 10, 61-64.

Hicks, L. H. (1956). An analysis of number-concept formation in the Rhesus monkey. Journal of Comparative \& Physiological Psychology, 49, 212-218.

Jobe, J. B., Mellgren, R. L., Feinberg, R. A., Littlejohn, R. L., * RigbY, R. L. (1977). Patterning, partial reinforcement, and N-length as a function of reinstatement of retrieval cues. Learning \& Motivation, 8, 77-97.

Meck, W. H., СhuRCh, R. M. (1983). A mode control model of counting and timing processes. Journal of Experimental Psychology: Animal Behavior Processes, 9, 320-334.

Rortblat, H. L., Polage, B., Scopatz, R. A. (1983). The representation of items in serial position. Animal Leaming \& Behavior, 11, 489-498.

TERRACE, H. S. (1986). A nonverbal organism's knowledge of ordinal position in a serial learning task. Journal of Experimental Psychology: Animal Behavior Processes, 12, 203-214.

(Manuscript received November 13, 1987; revision accepted for publication April 6, 1988.) 